\title{
Human Ocular Onchocerca lupi Infection Diagnosed in Norway
}

\author{
Tiril Sandell ${ }^{1,2 *}$, Anne Regine Skogen ${ }^{1}$, Tore Lier $^{3,4}$, Frank Olav Pettersen ${ }^{4}$ \\ ${ }^{1}$ Department of Ophthalmology, Oslo University Hospital, Oslo, Norway \\ ${ }^{2}$ Department of Ophtalmology, Drammen Sykehus, Vestre Viken HF, Drammen, Norway \\ ${ }^{3}$ Department of Infectious Diseases, University Hospital of North Norway, Tromsø, Norway \\ ${ }^{4}$ Regional Advisory Unit for Imported and Tropical Diseases, Department of Infectious Diseases, Ullevål, Oslo \\ University Hospital, Oslo, Norway
}

Corresponding Author: Tiril Sandell, MD, PhD, Department of Ophthalmology, Oslo University Hospital, Oslo, Norway. Tel: +47-92089791, Email: tiril.sandell@hotmail.com

Received May 31, 2020; Accepted September 21, 2020; Online Published November 15, 2020

\begin{abstract}
Introduction: Zoonotic Onchocerca lupi infection is a rare but emerging zoonotic disease affecting the eye, subcutaneous tissue and subdural cervical spine. Endemic areas of O. lupi are East and Central Europe, Turkey, Iran and the Northwest of the USA. Thirteen confirmed cases have been published in the world literature.

Case Presentation: We present a case of subconjunctival O. lupi infection in the left eye of a Norwegian male. The infection was most likely imported from Turkey. The diagnosis was verified by direct microscopy and molecular analyses with polymerase chain reaction (PCR) with subsequent sequencing. The worm did not contain microfilaria and was surgically removed. The patient was treated with tapering doses of topical steroids. At 6 months follow-up, he was asymptomatic.

Conclusion: Increasing numbers of $O$. lupi infections are reported and it is not clear whether the increasing number of infections is true or due to availability of novel diagnostic tools that correctly identify the cases. Humans cannot eliminate their exposure to vector-borne zoonotic diseases and increasing globalization increases the risk of infection. Both physicians and laboratory staff should be aware of the existence of zoonotic onchocerciasis in their countries.

Keywords: Onchocerca lupi, Zoonosis, Polymerase Chain Reaction, Zoonotic Disease
\end{abstract}

Citation: Sandell T, Skogen AR, Lier T, Pettersen FO. Human ocular Onchocerca lupi infection diagnosed in Norway. Int J Travel Med Glob Health. 2020;8(4):170-173. doi:10.34172/ijtmgh.2020.29

\section{Introduction}

Human zoonotic disease caused by Onchocerca lupi is rare but increasingly reported in the literature. Human infections with the nematode (round worm) O. lupi have been confirmed in 13 humans in Europe, Tunisia, Turkey, Iran and the USA.-3 The parasite's life cycle is not fully understood, but the parasite is transferred from animal to human through a vector, most likely the blackflies (Simuliidae). ${ }^{2}$ Most frequent presentation in animals and humans are ocular findings including variably painful subconjunctival swellings and nodules containing immature or mature worms affecting the eye or adjacent structures. Occasionally, extradural masses affecting the spinal cord have been seen in humans. ${ }^{1,4}$ Diagnosis is achieved by microscopy of excised adult female worms which exhibit a particular cuticular structure. Molecular analysis by polymerase chain reaction (PCR) with sequencing of the
PCR product can confirm the species. Treatment consists of surgical worm removal, sometimes accompanied by anthelmintic, antibiotic and anti-inflammatory therapy.

\section{Case Presentation}

A 54-year-old male of Turkish origin living in Norway since 1989 was presented to the Eye Clinic, Oslo University hospital (OUH) with a painful left red eye. The patient gave his written and oral consent to publish these data. The patient had been visiting family in Turkey on frequent occasions. The first complaint of a painful red eye came during a visit to Turkey in the late December 2018. On the first examination at the Eye Clinic, OUH, in February 2019, his left eye showed only slight injection of the conjunctiva with marked dilated vessels, but one week later we clearly identified a mobile, elevated subconjunctival mass superonasally. A white threadlike

Copyright $(\odot 2020$ The Author(s). This is an open-access article distributed under the terms of the Creative Commons Attribution License (http:// creativecommons.org/licenses/by/4.0), which permits unrestricted use, distribution, and reproduction in any medium, provided the original work is properly cited. 
structure was protruded from the lesion (Figure 1). The lesion was explored by slit lamp, and a $4-5 \mathrm{~cm}$ long structure thin as a sewing thread was extracted using fine forceps under local anesthetic. Later, the structure was identified as the adult roundworm O. lupi by microscopy and PCR with subsequent sequencing at the Public Health Agency and the National Veterinary Institute, Sweden and University of Zürich, Switzerland. Eosinophil blood cell count was normal. The laboratories saw no microfilariae within the worm on direct microscopy, hence our O. lupi was not considered a gravid female worm (Figure 2). The patient was seen at the Department of Infectious Diseases, OUH, and no extraocular clinical manifestations were noticed; thus, no further investigations or treatments were recommended. The patient was treated with topical steroids in tapering doses and seen at regular intervals. His symptoms were gradually resolved. At 6 months follow-up, the patient was symptom free and the only ophthalmological finding was slight hyperemic vessels in the area of previous worm removal.

\section{Discussion}

This is the fourteenth verified and published case of human O. lupi infection and the first patient diagnosed in Northern Europe to our knowledge. However, the infection was most likely imported from Turkey where O. lupi is endemic.

Onchocerca is a genus of nematodes (roundworms) with 34 different species, which primarily parasitize nonhuman hosts. ${ }^{5}$ The only well-established species known to cause human infection is $O$. volvulus, which causes onchocerciasis, also known as river blindness, primarily in Africa. ${ }^{6}$ In 1967, a new species infecting a wolf (Canis lupus cubanensis) was described in Russia and was named O. lupi. ${ }^{7}$ The taxonomy of this parasite is as follows: phylum Nematoda, order Spirurida, family Onchocercidae, genus Onchocerca, and species Onchocerca lupi. ${ }^{7}$ The first confirmed human cases of O. lupi were recorded in Turkey., 5,8

The life cycle of the parasite is not fully understood but believed to be similar to that of O. volvulus. According to Lok et $\mathrm{al},{ }^{10}$ Onchocerca spp. present two phases: one in blackflies (vector-immediate hosts) and another in definitive hosts (humans and animals). When vectors take a blood meal on an infected definitive host, these ingest microfilariae. The microfilariae develop to the third stage-infective larva (L3) in the mouth parts of the blackflies. This larval stage is infective to vertebrate definitive hosts. When infected blackflies take a subsequent blood meal from an uninfected vertebrate, the L3-larva is transmitted to a new vertebrate.

The most frequent clinical findings in animals and humans are, as in our case, unilateral or asymmetrical, variably painful subconjunctival swellings and nodules containing immature or mature worms affecting the eye and/or adjacent tissues accompanied by conjunctival hyperemia. ${ }^{2}$ Occasionally, subcutaneous nodules and masses affecting the spinal cord have been observed in humans., ${ }^{1,411}$ Differential diagnose of the ocular findings include orbital cellulitis, retro bulbar abscess, nodular episcleritis and periorbital neoplasia. When a roundworm is identified, the main differential diagnosis is Dirofilaria repens. Dirofilariasis affects the eye in similar ways

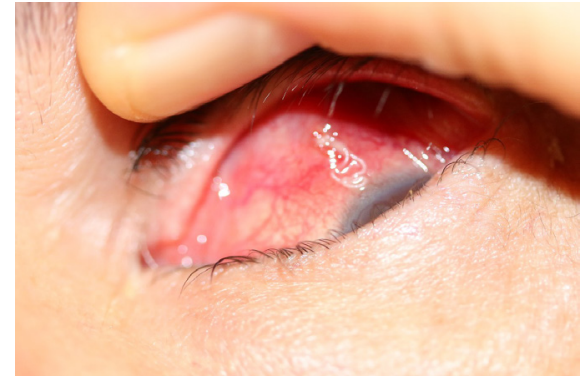

Figure 1. Clinical Presentation. Clinical presentation of the patient before worm removal. Roundworm in the superonasal quadrant of the left eye located partly subconjunctival, partly exposed. Conjunctival injection and hyperemia present.

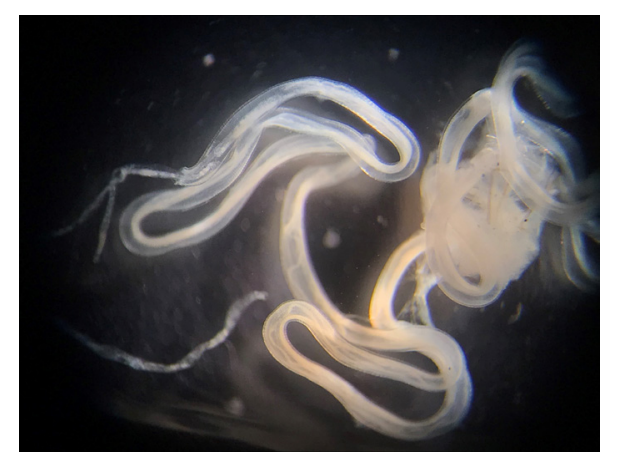

Figure 2. Loupe Photography of Extracted Onchocerca lupi. Loupe photography of the O. lupi specimen after extraction. A part of the worm broke off during the process.

as $O$. lupi and has been reposted to affect human eyes in more cases than O. lupi. ${ }^{12,13}$

The diagnosis is usually accidental when a filarial worm is excised from a nodule. The difficulties in recognizing $O$. lupi may arise from the fact that the infection is unknown to the examiner, whether the excised worm is immature, damaged during excision or fixed in Formalin, which may hamper molecular analysis. ${ }^{14}$ Diagnosis of O. lupi is achieved by microscopy of excised adult female worms which exhibit a particular cuticular structure. We found molecular analysis of the worm to be helpful in species determination. ${ }^{2}$ To the best of our knowledge, microfilariae have not been verified in human blood, but gravid female worms have been seen in humans. ${ }^{1}$ In animals, searching for microfilariae in blood smears has been shown to be unrewarding. ${ }^{15,16}$ Skin snips are commonly used but show low sensitivity in light infections. Blood eosinophilia is common but not always seen, as illustrated in our case. ${ }^{2}$ Diagnosis remains challenging in the absence of reliable non-invasive diagnostic tests. ${ }^{4}$

There are no evidence-based treatment algorithms for human O. lupi infection. ${ }^{1}$ In general, treatment consists of surgical worm removal and/or anthelminthic, antibiotic and anti-inflammatory therapy. ${ }^{2}$ Since data on the treatment of $O$. lupi is scarce, recommendations for medical therapy of $O$. lupi are based primarily on what is known about the treatment of 0 . Volvulus. ${ }^{17}$ In human onchocerciasis (river blindness), bacterial endosymbionts of the genus Wolbachia are believed to be essential for embryogenesis of microfilariae, and might therefore constitute an additional therapeutic target. Also, 
Wolbachia has been searched in $O$. lupi, ${ }^{18-22}$ and in nodules containing $O$. lupi nematodes removed from naturally infected dogs, Wolbachia-like endobacteria has been detected by electron microscopy. ${ }^{23}$ Doxycycline is known to make female worms of $O$. volvulus sterile by its antibacterial effect on the Wolbachia endosymbiont, which is required for reproduction and the long-term survival of the parasite. ${ }^{24}$ The anthelmintic drug ivermectin is microfilaricidal, so it may prevent symptomatic manifestations of river blindness caused by inflammatory reactions to dying microfilariae in subcutaneous tissue of the skin and the conjunctiva of the eye; it may also hinder transmission to blood-feeding blackflies. ${ }^{25}$

Since standardized treatment regimens for O. lupi infections are lacking, we opted to treat our patient with surgical removal of the parasite and tapering doses of topical steroids alone. Our worm did not contain microfilariae on direct microscopy, hence treatment with ivermectin was deemed unnecessary. On the other hand, one may argue that a single dose of ivermectin in this case would stop possible transmission of unrecognized microfilariae to susceptible humans or mammals if the patient was bitten by the appropriate vector while in Turkey. Postoperative Doxycycline treatment in order to sterilize the worm seemed inappropriate since the worm was removed in toto and there was no sign of multiple infection. In our opinion, based on available published literature, there is not enough data supporting the need for anti-helminthic or antibiotic therapy. Our patient recovered completely within six months post-surgery and after tapering out topical steroids.

\section{Conclusion}

Globalization with increased movement of people, food, livestock and pets combined with environmental and climate changes has led to the rapid expansion of emerging infections throughout the world. Human zoonotic infections with O. lupi have been increasingly recognized, yet they are still rare. However, the number of cases may be underestimated due to lack of knowledge and diagnostic tools. Furthermore, Dirofilaria, another filarial nematode, may have been wrongly identified as the cause of subconjunctival and periorbital masses such as the one seen in our patient. It is important to alert the scientific community, doctors, veterinarians, ophthalmologists, health service workers and the public about this poorly known emerging zoonotic disease which may also affect humans. Studies are needed to determine regular and potential definitive hosts and vectors of $O$. lupi in different areas in order to understand the reservoir, the transmission patterns and the seasonality of this zoonosis. Furthermore, research is needed to better define the burden of disease, identify targeted prevention strategies, and develop easily available diagnostic tools and therapeutic modalities.

\section{Authors' Contributions}

ARS and TS removed the worm and did the clinical follow up at the ophthalmological department. FOP advised, ARS and TS about the diagnostics of worm identification and contributed to the clinical follow up of the patient at the Department of Infectious Diseases, OUS. TL contributed to the molecular analysis of the worm and provided the lupe photograph of the worm (Figure 2). All authors discussed the case and contributed to the final manuscript.

\section{Conflict of Interest Disclosures}

No conflicting relationship exists for any author.

\section{Ethical Approval}

With regards to ethical approval code, in Norway this is not needed in case reports. Oral and written patient consent was given. Please see https://helseforskning.etikkom.no/

\section{Funding/Support}

None.

\section{References}

1. Cantey PT, Weeks J, Edwards M, et al. The emergence of zoonotic Onchocerca lupi Infection in the United States--a case-series. Clin Infect Dis. 2016;62(6):778-783. doi:10.1093/cid/civ983.

2. Grácio AJ, Richter J, Komnenou AT, Grácio MA. Onchocerciasis caused by Onchocerca lupi: an emerging zoonotic infection. Systematic review. Parasitol Res. 2015;114(7):2401-2413. doi:10.1007/s00436-015-4535-7.

3. Wesołowska M, Zając-Pytrus H, Masny A, et al. Onchocerca jakutensis ocular infection in Poland: a new vector-borne human health risk? Parasit Vectors. 2020;13(1):61. doi:10.1186/s13071020-3925-6.

4. Chen T, Moon K, deMello DE, Feiz-Erfan I, Theodore N, Bhardwaj RD. Case report of an epidural cervical Onchocerca lupi infection in a 13-year-old boy. J Neurosurg Pediatr. 2015;16(2):217-221. doi:10.3171/2014.12.peds14462.

5. Ilhan HD, Yaman A, Morishima Y, et al. Onchocerca lupi infection in Turkey: a unique case of a rare human parasite. Acta Parasitol. 2013;58(3):384-388. doi:10.2478/s11686-013-0152-8.

6. Basáñez MG, Pion SD, Churcher TS, Breitling LP, Little MP, Boussinesq M. River blindness: a success story under threat? PLoS Med. 2006;3(9):e371. doi:10.1371/journal.pmed.0030371.

7. Rodonaja TE. A new species of Nematode, Onchocerca lupi n. sp., from Canis lupus cubanensis. Soobshchenyia Akad Nauk Gruz SSR. 1967;45:715-719

8. Otranto D, Dantas-Torres F, Cebeci Z, et al. Human ocular filariasis: further evidence on the zoonotic role of Onchocerca lupi. Parasit Vectors. 2012;5:84. doi:10.1186/1756-3305-5-84.

9. Otranto D, Sakru N, Testini G, et al. Case report: first evidence of human zoonotic infection by Onchocerca lupi (Spirurida, Onchocercidae). Am J Trop Med Hyg. 2011;84(1):55-58. doi:10.4269/ajtmh.2011.10-0465.

10. Lok J, Walker E, GA S. Medical Entomology. Kluwer Academic Publisher; 2000:299-375.

11. Dudley RWR, Smith C, Dishop M, Mirsky D, Handler MH, Rao S. A cervical spine mass caused by Onchocerca lupi. Lancet. 2015;386(10001):1372. doi:10.1016/s0140-6736(14)62255-8.

12. Montesel A, Bendinelli A, Figus M, Posarelli C. There is a worm in my eye! Ocular dirofilariasis. Eur J Ophthalmol. 2019;29(5):NP5NP8. doi:10.1177/1120672118803519.

13. Pampiglione S, Rivasi F. Human dirofilariasis due to Dirofilaria (Nochtiella) repens: an update of world literature from 1995 to 2000. Parassitologia. 2000;42(3-4):231-254.

14. Sréter T, Széll Z, Egyed Z, Varga I. Subconjunctival zoonotic onchocerciasis in man: aberrant infection with Onchocerca lupi? Ann Trop Med Parasitol. 2002;96(5):497-502. doi:10.1179/000349802125001267.

15. Otranto D, Dantas-Torres F, Giannelli A, et al. Cutaneous distribution and circadian rhythm of Onchocerca lupi microfilariae in dogs. PLoS Negl Trop Dis. 2013;7(12):e2585. doi:10.1371/ 
journal.pntd.0002585.

16. Otranto D, Dantas-Torres F, Giannelli A, et al. Zoonotic Onchocerca lupi infection in dogs, Greece and Portugal, 20112012. Emerg Infect Dis. 2013;19(12):2000-2003. doi:10.3201/ eid1912.130264.

17. Cantey PT, Eberhard M, Weeks J, Swoboda S, Ostovar GA. Letter to the Editor: Onchocerca lupi infection. J Neurosurg Pediatr. 2016;17(1):118-119. doi:10.3171/2015.6.peds15344.

18. Sironi M, Bandi C, Sacchi L, Di Sacco B, Damiani G, Genchi C. Molecular evidence for a close relative of the arthropod endosymbiont Wolbachia in a filarial worm. Mol Biochem Parasitol. 1995;74(2):223-227. doi:10.1016/0166-6851(95)02494-8.

19. Bandi C, AndersonTJ, Genchi C, Blaxter ML. Phylogeny of Wolbachia in filarial nematodes. Proc Biol Sci. 1998;265(1413):2407-2413. doi:10.1098/rspb.1998.0591.

20. Taylor MJ, Hoerauf A. Wolbachia bacteria of filarial nematodes. Parasitol Today. 1999;15(11):437-442. doi:10.1016/s01694758(99)01533-1.

21. Hoerauf A, Volkmann L, Hamelmann C, et al. Endosymbiotic bacteria in worms as targets for a novel chemotherapy in filariasis. Lancet. 2000;355(9211):1242-1243. doi:10.1016/s0140-6736(00)02095-x.

22. Landmann F, Voronin D, Sullivan W, Taylor MJ. Anti-filarial activity of antibiotic therapy is due to extensive apoptosis after Wolbachia depletion from filarial nematodes. PLoS Pathog. 2011; 7(11):e1002351. doi:10.1371/journal.ppat.1002351.

23. Egyed Z, Sréter T, Széll Z, Nyirő G, Márialigeti K, Varga I. Molecular phylogenetic analysis of Onchocerca lupi and its Wolbachia endosymbiont. Vet Parasitol. 2002;108(2):153-161. doi:10.1016/ s0304-4017(02)00186-3.

24. Hoerauf A, Specht S, Büttner M, et al. Wolbachia endobacteria depletion by doxycycline as antifilarial therapy has macrofilaricidal activity in onchocerciasis: a randomized placebo-controlled study. Med Microbiol Immunol. 2008;197(3):295-311. doi:10.1007/ s00430-007-0062-1.

25. Basáñez MG, Pion SD, Boakes E, Filipe JA, Churcher TS, Boussinesq M. Effect of single-dose ivermectin on Onchocerca volvulus: a systematic review and meta-analysis. Lancet Infect Dis. 2008;8(5):310-322. doi:10.1016/s1473-3099(08)70099-9. 